\title{
Implications of gravitational waves for supersymmetric grand unification
}

\author{
So Chigusa $\odot,{ }^{1,2,3}$ Yuichiro Nakai, ${ }^{4}$ and Jiaming Zheng ${ }^{4}$ \\ ${ }^{1}$ Berkeley Center for Theoretical Physics, Department of Physics, University of California, \\ Berkeley, California 94720, USA \\ ${ }^{2}$ Theoretical Physics Group, Lawrence Berkeley National Laboratory, Berkeley, California 94720, USA \\ ${ }^{3}$ KEK Theory Center, IPNS, KEK, Tsukuba, Ibaraki 305-0801, Japan \\ ${ }^{4}$ Tsung-Dao Lee Institute and School of Physics and Astronomy, Shanghai Jiao Tong University, \\ 800 Dongchuan Road, Shanghai, 200240 China
}

(Received 22 November 2020; accepted 26 July 2021; published 27 August 2021)

\begin{abstract}
Supersymmetric grand unification based on $S O(10)$ is one of the most attractive paradigms in physics beyond the Standard Model. Inspired by the recent NANOGrav signal, we discuss the implications of detecting a stochastic gravitational wave background emitted by a network of cosmic strings for the $S O(10)$ grand unification. Starting from a minimal model with multiple steps of symmetry breaking, we show that it generally prefers a high intermediate scale above $10^{14} \mathrm{GeV}$ that is favored by observable primordial gravitational waves. The observed spectrum can potentially narrow the possible range of the cosmic string scale and restricts the unified couplings and the unification scale by requiring gauge coupling unification. As an indirect consequence of the high cosmic string scale, the monopole abundance places nontrivial constraints on the theory. These are complementary to the proton decay constraints and probe different facets of supersymmetric $S O(10)$ unification theories.
\end{abstract}

DOI: 10.1103/PhysRevD.104.035031

\section{INTRODUCTION}

The structure of the Standard Model (SM) matter sector, quarks and leptons, and the high-energy behavior of the SM gauge couplings strongly suggest that the three SM gauge groups $G_{\mathrm{SM}} \equiv S U(3)_{C} \times S U(2)_{L} \times U(1)_{Y}$ are unified at a high-energy scale [1]. The smallness of neutrino masses further indicates the existence of heavy right-handed neutrinos to realize the seesaw mechanism [2-4], which is a consequence of the grand unification based on $S O(10)[5,6]$. A large hierarchy of scales between the electroweak symmetry breaking (EWSB) and the grand unification is naturally stabilized by supersymmetry (SUSY). Amazingly, in the minimal supersymmetric SM (MSSM), the precise unification of the three SM gauge couplings is achieved. Moreover, with appropriate choices of Higgs representations to break the $S O(10)$, a $\mathbb{Z}_{2}$ subgroup known as the matter parity remains unbroken at all scales [7-10], while such parity is an ad hoc global symmetry in the MSSM. The matter parity forbids the rapid decay of protons and ensures the stability of the lightest supersymmetric particle which gives a viable candidate of

Published by the American Physical Society under the terms of the Creative Commons Attribution 4.0 International license. Further distribution of this work must maintain attribution to the author(s) and the published article's title, journal citation, and DOI. Funded by SCOAP ${ }^{3}$. the dark matter [11,12]. Therefore, the supersymmetric $S O(10)$ grand unified theory (GUT) is one of the most compelling frameworks of physics beyond the SM.

Since the grand unification is realized at a very high energy scale, its experimental test must be indirect. The most famous prediction of the GUT is the finite lifetime of the proton. The current lower limit from the SuperKamiokande experiment is $1.6 \times 10^{34}$ years [13] for the $p \rightarrow \pi^{0} e^{+}$decay mode and $5.9 \times 10^{33}$ years [14] for the $p \rightarrow K^{+} \bar{\nu}$ mode. In the near future, the limit is expected to be improved to $7.8 \times 10^{34}$ years $\left(3.2 \times 10^{34}\right.$ years $)$ for the $\pi^{0} e^{+}\left(K^{+} \bar{\nu}\right)$ mode at the Hyper-Kamiokande experiment [15]. Comparable sensitivities to the $p \rightarrow K^{+} \bar{\nu}$ decay of $6 \times 10^{34}$ years and $1.9 \times 10^{34}$ years are expected at the DUNE [16] and JUNO [17] experiments, respectively, with somewhat different time scales. Another hint may come from topological defects associated with GUT phase transitions. In particular, a network of cosmic strings can be produced and the matter parity renders them stable [18-20]. The cosmic strings form closed loops, shrink and lose energy via the emission of gravitational waves (GWs) $[21,22] .{ }^{1}$ Interestingly, a stochastic $\mathrm{GW}$ background

\footnotetext{
${ }^{1}$ Numerical simulations based on Nambu-Goto strings support this picture [23,24]. In the Abelian Higgs model, however, cosmic strings lose energy via particle productions, which suppress the GW production $[25,26]$. Recent simulations indicate that such particle productions are important only for very small loops [27].
} 
produced by cosmic strings stretches across a wide range of frequencies, and such a GW signal is one of the main targets in multifrequency GW astronomy and cosmology.

Recently, the NANOGrav collaboration has reported the first evidence of a stochastic GW background in pulsar timing data [28]. ${ }^{2}$ Although the signal is not conclusive, it can be well fitted by GWs from a network of cosmic strings because they can give a favored flat spectrum of frequencies in the GW energy density [32-35]. ${ }^{3}$ The energy density spectrum is proportional to $(G \mu)^{2}$ where $G$ is the Newton's constant and $\mu$ is the cosmic-string tension. The spectrum also depends on the loop size at the time of formation $\alpha$. This parameter has not been reliably estimated so far, but larger loop size $\alpha=0.01-0.1$ is typically favored by recent numerical simulations $[32,48,49]$. Conservatively, taking $\alpha=3 \times 10^{-4}, 5 \times 10^{-3}$, $1 \times 10^{-1}$, the NANOGrav signal can be fitted with $G \mu=1 \times 10^{-7}, 5 \times 10^{-9}, 1 \times 10^{-10}$, respectively [33]. The symmetry breaking scale associated with the formation of cosmic strings is related to the string tension, $v \sim 10^{16} \mathrm{GeV}\left(G \mu / 10^{-7}\right)^{1 / 2}$, whose precise coefficient is given by an $\mathcal{O}(1)$ group theory factor. Then, the NANOGrav signal indicates the symmetry breaking scale is in the range, $10^{14} \mathrm{GeV} \lesssim v \lesssim 10^{16} \mathrm{GeV}$.

The interpretation of the NANOGrav signal with cosmic strings from a GUT phase transition indicates the existence of intermediate steps [50-54] in the breaking of $S O(10) \rightarrow G_{\mathrm{SM}} \times M$ (with $M$ the matter parity) because the $S O(10)$ breaking also predicts monopoles that may overclose the universe. In a natural cosmologically safe scenario, monopoles are only produced during symmetry breaking at high energy scales and get diluted away by inflation afterward. The remaining intermediate gauge group is finally broken to $G_{\mathrm{SM}}$ at a lower scale $v \gtrsim 10^{14} \mathrm{GeV}$ where cosmic strings emitting GWs are formed.

In this paper, we study the consequences of such intermediate scales in a supersymmetric $S O(10)$ theory. The particles with masses below the unification scale largely alter the renormalization group (RG) evolution of the gauge couplings from that in MSSM. Interestingly, it turns out that the unification of gauge couplings enforces high intermediate scales that are needed by the cosmic string interpretation of the NANOGrav result. Thus, the

\footnotetext{
${ }^{2}$ The NANOGrav result is apparently in tension with the previous results by other pulsar timing arrays EPTA [29] and PPTA [30]. However, it is argued in [28] that this tension is the result of the improved treatment of the intrinsic pulsar red noise. A recent solution invoking partially inflated cosmic string during inflation is given in [31].

${ }^{3}$ Other interpretations of sources to generate GWs to explain the NANOGrav signal include phase transitions [36-40], primordial black hole formation [41-46] and dynamics of axionlike particles $[38,47]$.
}

observation of cosmic string GW does not only imply the existence of an intermediate scale but also strongly motivates supersymmetric $S O(10)$ as the unified theory of gauge interactions.

The remainder of this paper is organized as follows. In Sec. II, we summarize the models and patterns of gauge symmetry breaking considered in this paper. In Sec. III, we show the calculation of the running of gauge coupling constants and the resulting constraint from the gauge coupling unification. Section IV and Sec. V are devoted to the calculation of the proton decay rate and the monopole density, respectively, with showing the resulting constraints. We conclude and give some discussions of our results in Sec. VI.

\section{MODELS}

$S O(10)$ is a rank 5 simple group and contains an additional $U(1)$ factor in addition to $G_{\mathrm{SM}}$ of rank 4 . The minimal choices of Higgs representations to break the $U(1)$ in a SUSY model are $\mathbf{1 6}+\overline{\mathbf{1 6}}$ or $\mathbf{1 2 6}+\overline{\mathbf{1 2 6}}$. We will stick to the latter choice as it preserves the matter parity $M \equiv(-1)^{3(B-L)}$ where $B$ and $L$ are baryon and lepton numbers. Vacuum expectation values (VEVs) of SM singlets in $\mathbf{1 2 6}+\overline{\mathbf{1 2 6}}$ leave the $S U(5)$ subgroup unbroken and more Higgs fields are needed. The minimal Higgs fields that break $S O(10)$ to $G_{\mathrm{SM}} \times M$ are then $\mathbf{4 5}+\mathbf{5 4}+\mathbf{1 2 6}+\overline{\mathbf{1 2 6}}^{4}$ and we will focus on this choice throughout the paper. The two Higgs doublets $H_{u}, H_{d}$ in the MSSM are chosen to be bidoublets in $\mathbf{1 0}$ for the simplicity of analysis.

To summarize, we consider SUSY SO(10) models with the following set of heavy Higgs fields [57],

$S=54, \quad A=45, \quad \Sigma=126, \quad \bar{\Sigma}=\overline{\mathbf{1 2 6}}$.

The most general renormalizable superpotential of the Higgs sector is

$$
\begin{aligned}
W_{H}= & \frac{m_{S}}{2} \operatorname{Tr} S^{2}+\frac{\lambda_{S}}{3} \operatorname{Tr} S^{3}+\frac{m_{A}}{2} \operatorname{Tr} A^{2}+\lambda \operatorname{Tr} A^{2} S \\
& +m_{\Sigma} \Sigma \bar{\Sigma}+\eta_{S} \Sigma^{2} S+\bar{\eta}_{S} \bar{\Sigma}^{2} S+\eta_{A} \Sigma \bar{\Sigma} A+\cdots,
\end{aligned}
$$

where we have omitted the terms with $\mathbf{1 0}$. We find minima of the Higgs potential by solving the $F$ - and $D$-flat conditions. We express the VEVs of $G_{\mathrm{SM}}$ singlet fields in terms of representations under the Pati-Salam group $G_{422} \equiv S U(4)_{C} \times S U(2)_{L} \times S U(2)_{R}$ to which they belong:

\footnotetext{
"The choice of $\mathbf{4 5}+\mathbf{5 4}+\mathbf{1 2 6}+\overline{\mathbf{1 2 6}}$ is "minimal" in the number of components. Another popular choice is $\mathbf{2 1 0}+\mathbf{1 2 6}+$ $\overline{\mathbf{1 2 6}}[55,56]$ which leads to the "minimal" number of parameters in the general Lagrangian.
} 
TABLE I. The summary of patterns of VEVs at local minima of the potential that lead to a subgroup $H$ of $S O(10)(\checkmark$ denotes a nonzero VEV). Stars in the first column indicate that the symmetry breaking $H \rightarrow G_{\mathrm{SM}} \times M$ is associated with the formation of cosmic strings. A circle in the first column indicates the formation of monopoles and cosmic strings at the same time, which is not of our interest.

\begin{tabular}{lcccc}
\hline \hline$H$ & $s$ & $a$ & $b$ & $\sigma$ \\
\hline$S O(10)$ & & & & \\
$\circ S U(4) \times S U(2) \times S U(2)$ & $\checkmark$ & & & \\
$\star S U(3) \times S U(2) \times S U(2) \times U(1)$ & $\checkmark$ & $\checkmark$ & & \\
$\star S U(4) \times S U(2) \times U(1)$ & $\checkmark$ & & $\checkmark$ & \\
$S U(5) \times M$ & & $\checkmark$ & $\checkmark$ & $\checkmark$ \\
$G_{\mathrm{SM}} \times M$ & $\checkmark$ & $\checkmark$ & $\checkmark$ & $\checkmark$ \\
\hline \hline
\end{tabular}

$$
\begin{aligned}
& s=\langle S(1,1,1)\rangle, \quad a=\langle A(15,1,1)\rangle, \quad b=\langle A(1,1,3)\rangle, \\
& \sigma=\langle\Sigma(10,1,3)\rangle, \quad \bar{\sigma}=\langle\bar{\Sigma}(\overline{10}, 1,3)\rangle .
\end{aligned}
$$

Patterns of VEVs which satisfy the minimum condition and lead to a subgroup $H$ of $S O(10)$ are summarized in Table I. Note that the $D$-flatness sets $|\sigma|=|\bar{\sigma}|$.

Motivated by the result of NANOGrav, we focus on symmetry breaking patterns where cosmic strings form at an intermediate scale $M_{R} \sim 10^{14-16} \mathrm{GeV}$. As indicated by stars in the first column of Table I, there are two possible choices of $H$ whose breaking into $G_{\mathrm{SM}} \times M$ is associated with the cosmic string formation without accompanying monopoles: $G_{3221} \equiv S U(3)_{C} \times S U(2)_{L} \times$ $S U(2)_{R} \times U(1)_{B-L}$ and $G_{421} \equiv S U(4)_{C} \times S U(2)_{L} \times U(1)_{R}$. When there is a hierarchy between VEVs of Eq. (3), the multistep breaking of $S O(10)$ with at least one intermediate scale is achieved. The simplest possibility is the breaking with one intermediate scale, i.e., $S O(10) \rightarrow H \rightarrow G_{\mathrm{SM}} \times M$, where the breaking scale of $H$ is identified as $M_{R}$. A detailed analysis of this possibility is given in the Appendix, where we show that it is difficult to construct a viable model that survives collider constraints of the SUSY particle search. Thus, in the following discussion, we focus on models with two intermediate scales, with the lower scale identified as $M_{R}$, and show that $M_{R} \gtrsim 10^{14} \mathrm{GeV}$ is compatible with the unification of gauge couplings. There are only two possible breaking patterns that lead to surviving cosmic strings, $S O(10) \rightarrow G_{422} \rightarrow H \rightarrow$ $G_{\mathrm{SM}} \times M$ with $H=G_{3221}$ (model A) or $H=G_{421}$ (model B).

The model A is obtained through the hierarchical choice of VEVs given by $|s| \gg|a| \gg|\sigma|$, while $|b| \sim|\sigma|^{2} /|s| \ll$ $|\sigma|$ is ensured from the minimization condition of the potential. We can define the unification scale $M_{X}$, the PatiSalam breaking scale $M_{C}$, and the $G_{3221} \rightarrow G_{\mathrm{SM}} \times M$ breaking scale $M_{R}$ through $M_{X} \sim|s|, M_{C} \sim|a|$, and $M_{R} \sim|\sigma|$, respectively. We summarize the mass spectrum of all the components of $S, A, \Sigma$, and $\bar{\Sigma}$ Higgs fields in
TABLE II. The mass spectrum of $S, A, \Sigma$, and $\bar{\Sigma}$ Higgs fields in the model A. $(1,1)_{0, \pm 1, \pm 2}$ denotes charges under the SM gauge groups $G_{\mathrm{SM}} \equiv S U(3)_{C} \times S U(2)_{L} \times U(1)_{Y}$.

\begin{tabular}{lc}
\hline \hline States & Mass scale \\
\hline$\Sigma(\overline{10}, 3,1), \bar{\Sigma}(10,3,1)$ & $M_{C}$ \\
Color triplets and sextets of $\Sigma(10,1,3)$, & $M_{C}$ \\
$\bar{\Sigma}(\overline{10}, 1,3)$ & \\
Color triplets of $A(15,1,1)$ & $M_{C}$ \\
$(1,1)_{0},(1,1)_{ \pm 1}$ from $\Sigma(10,1,3)$, & $M_{R}$ \\
$\bar{\Sigma}(\overline{10}, 1,3)$ & $M_{1} \equiv \operatorname{Max}\left[\frac{M_{R}^{2}}{M_{C}}, \frac{M_{C}^{2}}{M_{X}}\right]$ \\
A color octet and a singlet of & $M_{2} \equiv M_{R}^{2} / M_{X}$ \\
$A(15,1,1)$ & \\
$(1,1)_{0},(1,1)_{ \pm 2}$ from $\Sigma(10,1,3)$, & $M_{X}$ \\
$\bar{\Sigma}(\overline{10}, 1,3)$ & \\
All the other components & \\
\hline \hline
\end{tabular}

Table II. ${ }^{5}$ Note that some states have masses different from the scales $M_{X, C, R}$. The mass scale $M_{2}$ is always smaller than $M_{1, R}$, while the order of $M_{1}$ and $M_{R}$ is not fixed in general. When we perform multiple steps of RGE running, we assume all particles at an energy scale $Q$ share the same mass $Q$. Generally, their masses depend on coupling constants and the nonuniform spectrum generates a threshold correction [58,59]. We discuss this point in the Appendix.

The model B is obtained when $|s| \gg|b| \gg|\sigma|$ and $|a| \sim|\sigma|^{2} /|s| \ll|\sigma|$. However, it turns out that typically the unification scale is lower in this model than that in the model A. As a result, the current constraint from the proton decay rate is more severe and only a tiny region remains unconstrained. Thus, we will not consider this possibility and focus on the model $\mathrm{A}$ in the following discussion.

Finally, some comments on the fermion masses are in order. It is well known that if the MSSM higgs doublets $H_{u}$ and $H_{d}$ are embedded only in the $\mathbf{1 0}$ (or $\overline{\mathbf{1 2 6}}$ ), the SM fermion masses at the GUT scale will be given by $m_{\ell}=$ $m_{d}$ (or $m_{\ell}=-3 m_{d}$ ) for all three generations [60], which is in tension with the measured light fermion masses at low scales. However, these relations of masses are altered by loop effects and higher-dimensional operators suppressed by the Planck scale [61-63]. In a renormalizable setup, the MSSM Higgs doublets can be taken as linear combinations of doublets in $\mathbf{1 6}$ or $\mathbf{1 2 6}$ which renders the mass relation at the GUT scale arbitrary. However, it is impossible to mix the two doublets in the minimal choice of $\mathbf{4 5}+\mathbf{5 4}+\mathbf{1 0}+\mathbf{1 2 6}+\overline{\mathbf{1 2 6}}$ for the Higgs sector with a renormalizable superpotential [64]. One economical solution is to introduce a $\mathbf{1 2 0}$ so that $\mathbf{1 0}$ mixes

\footnotetext{
${ }^{5}$ Note that there can be mixing among the Higgs doublets in $10, \Sigma$, and $\bar{\Sigma}$, and two of them obtain light masses to be MSSM Higgs doublets. The other Higgs doublets obtain masses of $\mathcal{O}\left(M_{X}\right)$, which are summarized in the last column of Table II.
} 
with $\overline{\mathbf{1 2 6}}$ by the superpotential terms 45.10 .120 and 45.126.120 [64]. ${ }^{6}$ Since there are several approaches toward the SM fermion spectrum, we assume the MSSM Higgs doublets lie mostly in $\mathbf{1 0}$ for the simplicity of the RGE analysis, and leave it open to specify the mechanism of realizing the measured fermion masses.

\section{GAUGE COUPLING UNIFICATION}

Let us now consider the RG evolution of the gauge couplings from the unification scale $M_{X}$ to the weak scale $M_{Z}$ experiencing $S O(10) \rightarrow G_{422} \rightarrow G_{3221} \rightarrow G_{\mathrm{SM}} \times M$. We assume the mass scale of supersymmetric particles $M_{S}$ is smaller than $M_{2}$. Then, the evolution of the three SM gauge couplings $\alpha_{i} \equiv g_{i}^{2} / 4 \pi(i=1,2,3)$ is governed by the SM and MSSM beta functions up to $M_{S}$ and $M_{2}$, respectively, while light states from GUT Higgses contribute to the running from $M_{2}$ to $M_{R}$. At the $G_{3221}$ breaking scale $M_{R}$, the gauge couplings of $S U(2)_{R}$ and $U(1)_{B-L}$, denoted as $\alpha_{2 R}$ and $\alpha_{B-L}$, respectively, are matched to that of the SM hypercharge with

$$
\frac{3}{5} \alpha_{2 R}^{-1}\left(M_{R}\right)+\frac{2}{5} \alpha_{B-L}^{-1}\left(M_{R}\right)=\alpha_{1}^{-1}\left(M_{R}\right),
$$

and $\alpha_{2 R}$ and $\alpha_{B-L}$ run further up to the $G_{422}$ breaking scale $M_{C}$. The matching condition at $M_{C}$ is

$$
\alpha_{4}\left(M_{C}\right)=\alpha_{3}\left(M_{C}\right)=\alpha_{B-L}\left(M_{C}\right),
$$

where $\alpha_{4}$ denotes the $S U(4)_{C}$ gauge coupling. Finally, $\alpha_{4}$, $\alpha_{2}$, and $\alpha_{2 R}$ run to $M_{X}$ and unify into a unique value $\alpha_{U}$. At the one-loop level, the relationship between the gauge coupling constants $\alpha_{i}$ at $M_{Z}$ and $\alpha_{U}$ is described by

$$
\begin{aligned}
\frac{2 \pi}{\alpha_{i}\left(M_{Z}\right)}= & \frac{2 \pi}{\alpha_{U}}+\left[b_{i}^{(1)} \ln \frac{M_{S}}{M_{Z}}+b_{i}^{(2)} \ln \frac{M_{2}}{M_{S}}+b_{i}^{(3)} \ln \frac{M_{1}}{M_{2}}\right. \\
& \left.+b_{i}^{(4)} \ln \frac{M_{R}}{M_{1}}+b_{i}^{(5)} \ln \frac{M_{C}}{M_{R}}+b_{i}^{(6)} \ln \frac{M_{X}}{M_{C}}\right],
\end{aligned}
$$

for $M_{1}<M_{R}$, while the role of $M_{1}$ and $M_{R}$ should be interchanged if $M_{1}>M_{R}$. The coefficients $b_{i}^{(k)}$ are summarized in Table III. In particular, $b_{i}^{(3,4,5)}$ are written in a compact form within $M_{2}<Q<M_{C}$ with thresholds at $M_{1}$ and $M_{R}$ represented by step functions $\theta_{1}$ and $\theta_{R}$. We solve these three equations in terms of the three parameters $M_{X, C, R}$ and obtain a set of solutions as functions of $M_{S}$ and $\alpha_{U}$. In our numerical analysis, we use the two-loop RG equations $[65,66]$ below $M_{R}$ while including only the oneloop contributions from the light states.

\footnotetext{
${ }^{6}$ In addition, there are four additional scalar doublets in the $\mathbf{1 2 0}$ chiral multiplet that can be part of the MSSM Higgs doublets.

${ }^{7}$ The convention for the charge of $U(1)_{B-L}$ is $q_{B-L}=\frac{3}{8}(B-L)$.
}

TABLE III. The coefficients of the RG equations of the gauge coupling constants for each energy range in the model A. Note that $\theta_{R} \equiv \Theta\left(Q-M_{R}\right)$ and $\theta_{1} \equiv \Theta\left(Q-M_{1}\right)$ with $\Theta$ being the Heaviside step function.

\begin{tabular}{lcccc}
\hline \hline Energy range & $b_{1}^{(k)}$ & $b_{2}^{(k)}$ & $b_{3}^{(k)}$ & $(k)$ \\
\hline$M_{Z}<Q<M_{S}$ & $41 / 10$ & $-19 / 6$ & -7 & $(1)$ \\
$M_{S}<Q<M_{2}$ & $33 / 5$ & 1 & -3 & $(2)$ \\
$M_{2}<Q<M_{C}$ & $57 / 5-12 / 5 \theta_{R}$ & 1 & $-3+3 \theta_{1}$ & $(3),(4),(5)$ \\
$M_{C}<Q<M_{X}$ & $191 / 5$ & 41 & 34 & $(6)$ \\
\hline \hline
\end{tabular}

Figure 1 shows contours of the intermediate scale $M_{R}$ as a function of $M_{S}$ and $\alpha_{U}$. The results in the left and the middle panels are obtained with universal soft masses, while the result in the right panel is obtained for a split spectrum with gaugino masses fixed at $\mathcal{O}(1) \mathrm{TeV}$ with other superpartners of SM particles at $M_{S}$. In the middle panel, we take account of typical sizes of GUT-scale threshold corrections (see Appendix for the details). The colored contours correspond to the choices $M_{R}=$ $4 \times 10^{14} \mathrm{GeV}$ (red), $10^{15} \mathrm{GeV}$ (green), and $4 \times 10^{15} \mathrm{GeV}$ (blue), while the solid and dotted lines correspond to $\tan \beta=2$ and 50, respectively. The dependence on $\tan \beta$ comes from the two-loop contribution to RG equations from Yukawa couplings. Note that in a concrete model, $\tan \beta$ is constrained by the fermion mass spectrum. Nevertheless, it has little impact on RG running and we will use $\tan \beta=2$ as a representative value. The lower gray region is excluded by either $M_{C}<M_{R}$ or $M_{X}<M_{C}$. In the former case, monopoles are produced alongside cosmic strings. In the latter case, unification cannot be achieved. All the viable parameter space in the figure leads to the unification scale $M_{X}$ of $\mathcal{O}\left(10^{16}\right) \mathrm{GeV}$ or above.

In passing, we note that in addition to the intermediate scale states listed in Table II, the $(\mathbf{1 5}, \mathbf{2}, \mathbf{2})$ multiplet in $\overline{\mathbf{1 2 6}}$ can also lie at the scale $M_{C}$ if they obtain a vev that breaks the electroweak symmetry. This can be the case if $\overline{\mathbf{1 2 6}}$ mixes with $\mathbf{1 0}$ by an additional $\mathbf{1 2 0}$ for the SM fermion masses [64]. Fortunately, this only slightly changes our result of symmetry breaking scales since its contribution to the $S U(4)_{C}$ and $S U(2)_{L, R}$ beta functions satisfy $b_{4} \approx b_{2 L}=b_{2 R}$. This effect is overwhelmed by uncertainties we further discuss in the remaining of this paper.

\section{PROTON DECAY}

As is often the case for SUSY GUT models, the proton lifetime imposes severe constraints on the model parameter space. The proton decay can be induced by the dimension- 5 operators from the colored Higgs exchange [67-69]. Contrary to SUSY SU(5) models, the existence of several colored Higgs multiplets in the present model leads to various decay branches such as $p \rightarrow K^{0} \ell^{+}$as well as the popular $p \rightarrow K^{+} \bar{\nu}$ mode [70,71]. However, since the current constraints on such unusual decay modes are generally 

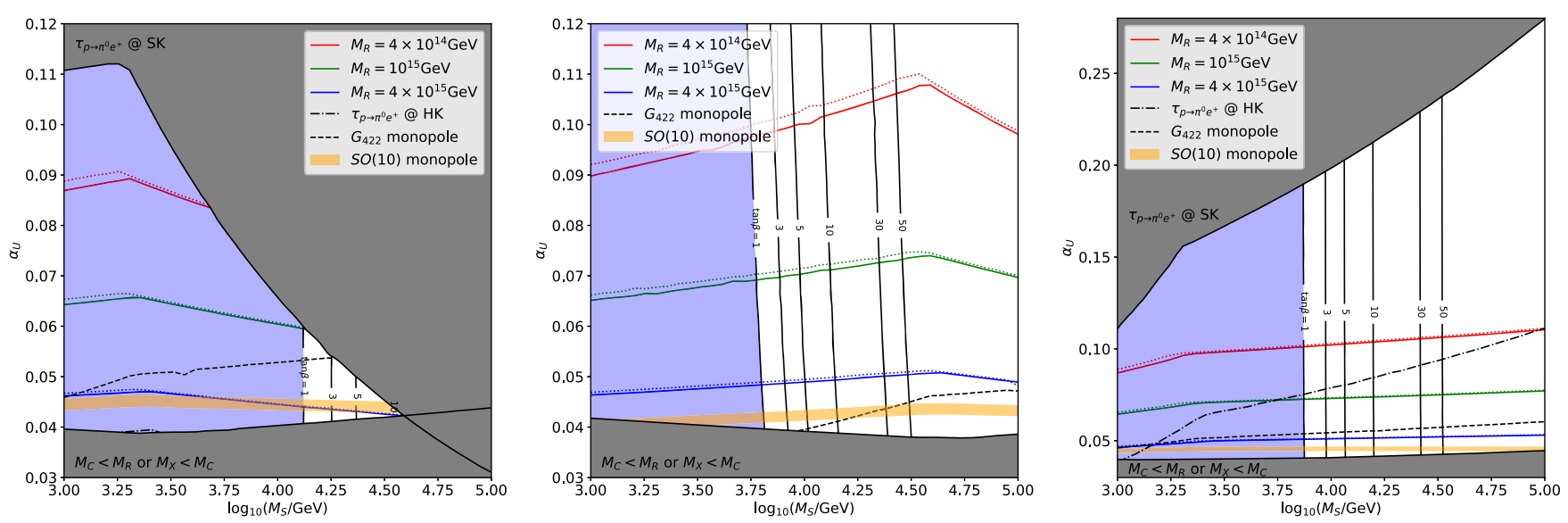

FIG. 1. Contour plot of $M_{R}$ as a function of $M_{S}$ and $\alpha_{U}$. Left panel: universal soft masses without threshold correction. Middle panel: universal soft masses with typical threshold corrections at the GUT-scale. Right panel: split SUSY spectrum with masses of gauginos fixed at $\mathcal{O}(1) \mathrm{TeV}$ and without threshold corrections. The red, green, and blue lines correspond to $M_{R}=4 \times 10^{14} \mathrm{GeV}, 10^{15} \mathrm{GeV}$, $4 \times 10^{15} \mathrm{GeV}$, respectively, with $\tan \beta=2$ (solid) and 50 (dotted). The lower gray region is excluded for either $M_{C}<M_{R}$ (abundant monopole) or $M_{X}<M_{C}$ (unachievable unification). The upper gray and the left blue regions are excluded by the current lower bound on the proton partial lifetime $\tau_{p \rightarrow \pi^{0} e^{+}}$and $\tau_{p \rightarrow K^{+} \bar{\nu}}$, respectively. The regions to the left of vertical lines are excluded by $\tau_{p \rightarrow K^{+} \bar{\nu}}$ depending on the choice of $\tan \beta=1,3,5,10,30,50$ from left to right. The dot-dashed line represents the future sensitivity on $\tau_{p \rightarrow \pi^{0} e^{+}}$at the Hyper Kamiokande. The region below the dashed line leads to excessive abundance of intermediate scale monopoles. The region below the orange band is excluded by catalyzed nucleon decays inside compact stars by GUT monopoles.

weaker than that on the $p \rightarrow K^{+} \bar{\nu}$ mode, we focus on the latter. The proton lifetime is roughly given by [72]

$\tau_{p \rightarrow K^{+} \bar{\nu}} \sim 10^{35} \mathrm{yrs} \times \sin ^{4} 2 \beta\left(\frac{M_{S}}{10^{5} \mathrm{GeV}}\right)^{2}\left(\frac{M_{H_{C}}}{10^{16} \mathrm{GeV}}\right)^{2}$.

In the following estimation, we will simply approximate the colored Higgs mass $M_{H_{C}} \approx M_{X}$.

The dimension-5 proton decay rate is model dependent and Eq. (7) should be regarded as a rough estimate with large theoretical uncertainty. First, the size of the coupling between a colored Higgs and SM fermions is determined by the Yukawa coupling at the GUT scale, which is a source of the $\tan \beta$ dependence. However, values of Yukawa couplings highly depend on the structure of the Yukawa sector in terms of $S O(10)$ superfields, while we use typical values of Yukawa couplings obtained by running the MSSM RG equations in the estimation. Secondly, the intermediate scales alter the running of Wilson coefficients from those in SUSY $S U(5)$ models [72] with which we perform our calculation. However, this effect is not significant due to the proximity between $M_{R}$ and $M_{X}$, and is overwhelmed by the uncertainty in the mass spectrum of sfermions. Furthermore, Eq. (7) depends on the squared masses $M_{H_{c}}^{2}$ of the colored Higgses in 10. After setting the doublets to the weak scale, $M_{H_{c}}$ is a linear function of the vev $s \sim \mathcal{O}\left(M_{X}\right)$ and the dimensionless coupling of the superpotential 10.10.54. If the dimensionless couplings are taken to be $\mathcal{O}(1)$, the color triplet mass $M_{H_{c}}$ can also vary by an
$\mathcal{O}(1)$ factor. As a whole, the above estimation of the proton decay rate induced by the colored Higgses is expected to bear uncertainty of $\mathcal{O}(1 \sim 10)$ in each direction and can be worse for extremely small or large couplings in the superpotential.

Another important contribution to proton decay comes from dimension- 6 operators induced by the heavy gauge boson exchange $[73,74]$. Relevant gauge bosons are those that transform under $G_{\mathrm{SM}}$ as $(3,2)_{5 / 6}$ and $(3,2)_{-1 / 6}$. Compared with SUSY SU(5) models, the number of gauge bosons that contribute to the proton decay doubles, and the proton decay width to the most important decay mode, $p \rightarrow \pi^{0} e^{+}$, increases. The proton lifetime is given by [75]

$$
\tau_{p \rightarrow \pi^{0} e^{+}} \sim 5 \times 10^{34} \mathrm{yrs} \times\left(\frac{0.04}{\alpha_{U}}\right)^{2}\left(\frac{M_{X}}{10^{16} \mathrm{GeV}}\right)^{4},
$$

where we simply assumed that all the relevant dimension- 6 operators are mediated by a gauge boson with mass $M_{X}$ for a model-independent analysis. Note that the proton lifetime is highly sensitive to the gauge boson mass as can be seen from the fourth-power dependence in Eq. (8), and thus the model dependent analysis of the GUT spectrum will be needed for a more precise estimation, which is however out of the scope of this paper. In the evaluation, we have used the RGE factor of Wilson coefficients valid for the MSSM running up to $M_{X}$ [76], which can be slightly modified due to the difference of RGEs above $M_{R}$.

In Fig. 1, we show the current lower limit on the proton lifetime from the Super-Kamiokande experiment, $\tau_{p \rightarrow K^{+} \bar{\nu}}>5.9 \times 10^{33}$ yrs [14], with black vertical lines. 
The lines correspond to $\tan \beta=1,3,5,10,30$ and 50 from left to right. The region to the left of each line with smaller $M_{S}$ is excluded for each $\tan \beta$. Therefore, the left blue region, whose right boundary corresponds to $\tan \beta=1$, is excluded for all $\tan \beta \geq 1$. The upper gray region is the parameter space that is excluded by the current limit of $\tau_{p \rightarrow \pi^{0} e^{+}}>1.6 \times 10^{34} \mathrm{yrs}$ [13], while the dot-dashed line represents the prospect of Hyper Kamiokande [15] as an example of the future experiments. Note that the GUT scale is generally large in the middle panel of Fig. 1. As a result, the constraint from $p \rightarrow \pi^{0} e^{+}$is absent in this panel.

\section{MONOPOLE DENSITY}

In a series of phase transitions, $S O(10) \rightarrow G_{422} \rightarrow$ $G_{3221} \rightarrow G_{\mathrm{SM}} \times M$, we assume that inflation occurs before the $G_{3221} \rightarrow G_{\mathrm{SM}} \times M$ phase transition and the reheating temperature $T_{R}$ is above the critical temperature of this phase transition so that cosmic strings that emit $\mathrm{GW}$ are populated in the Universe. On the other hand, the breaking of $G_{422} \rightarrow$ $G_{3221}$ generates intermediate scale monopoles with mass $m_{m} \simeq M_{C} / \alpha_{4}$ [77,78]. To avoid the overclosure of the Universe, we require $T_{R}<M_{C}$ so that the $G_{422}$ symmetry is not restored during the reheating and monopoles are not generated by the Kibble mechanism [18]. However, when $T_{R}$ is not much lower than $M_{C}$, monopoles may still be produced through annihilation of particles in the thermal bath with a suppressed rate [79,80]. For $m_{m} / T_{R} \lesssim 20$, the monopole is thermalized and its relic density overcloses the Universe. For $m_{m} / T_{R} \gtrsim 20$, the monopole is produced in out-of-equilibrium with a final density,

$$
\frac{n_{m}}{n_{\gamma}} \simeq 3 \times 10^{3}\left(\frac{m_{m}}{T_{R}}\right)^{3} e^{-\frac{2 m_{m}}{T_{R}}},
$$

where $n_{\gamma}$ is the photon number density. Such monopole density is constrained in several ways. The dark matter cannot be solely made of monopoles because the local monopole density would be in severe contradiction with the null result of monopole searches such as MACRO [81]. We can then require $\Omega_{m} \ll \Omega_{M}$, where $\Omega_{m}$ and $\Omega_{M}$ are the monopole and dark matter densities normalized by the critical density of the Universe. This sets $m_{m} / T_{R} \gtrsim 40$ for $\sim 1 \mathrm{GeV}$. Another equally strong constraint comes solely from the direct search of monopoles by MACRO. The intermediate scale monopoles can be accelerated by the galactic magnetic field $[82,83]$ to a high velocity of order $10^{-3} \sqrt{10^{17} \mathrm{GeV} / m_{m}}$. Thus, they are not bounded to galaxies and we assume them to be uniformly distributed in the Universe, with a flux near the Earth,

$F_{m} \simeq 3.7 \times 10^{-17} \mathrm{~cm}^{-2} \mathrm{~s}^{-1} \times\left(\frac{n_{m} / n_{\gamma}}{3 \times 10^{-27}}\right)\left(\frac{v_{m}}{300 \mathrm{kms}^{-1}}\right)$, where $v_{m}$ is the relative velocity between the monopole and the Earth. The MACRO monopole search puts a constraint on the flux, $F_{m} \lesssim 1.8 \times 10^{-15} \mathrm{~cm}^{-2} \mathrm{~s}^{-1}$, which again sets the limit $m_{m} / T_{R} \gtrsim 40$. Assuming the critical temperature $T_{c}$ of the $G_{3221} \rightarrow G_{\mathrm{SM}} \times M$ phase transition is around the symmetry breaking scale, $T_{R} \gtrsim T_{c} \sim M_{R}$ is required for the production of cosmic strings. We thus find a constraint on the hierarchy between the two intermediate scales, $M_{C} / T_{R} \gtrsim M_{C} / M_{R} \gtrsim 40 \alpha_{4}$. This constraint is shown in Fig. 1 with dashed lines; the regions below the dashed lines are constrained.

The constraints above are trivial for GUT monopoles since their density is more exponentially suppressed. However, GUT monopoles catalyze baryon number violation processes $[84,85]^{8}$ in compact stars and increase their luminosity. Various stringent bounds have been obtained in the literature [83]. The observation of old white dwarfs constrains $F_{m}^{\prime} \lesssim 10^{-18} \sim 10^{-21} \mathrm{~cm}^{-2} \mathrm{~s}^{-1} \quad[86,87]$. The measured x-ray fluxes of old nearby pulsars constrain $F_{m}^{\prime} \lesssim$ $10^{-22} \sim 10^{-28} \mathrm{~cm}^{-2} \mathrm{~s}^{-1}$ [88-92]. We note that the bounds are subjected to large astrophysical uncertainties. In particular, the quoted stronger or weaker end of the upper bound correspond to whether or not the monopoles captured in the progenitor star can be kept in the compact star. To apply these bounds, we note that the mass of the GUT monopole is typically $M_{m} \simeq M_{X} / \alpha_{U} \sim 10^{17} \mathrm{GeV}$. Such heavy monopoles cannot be efficiently accelerated by the galactic magnetic field and are gravitationally bounded to galaxies with enhanced density. We approximate the local monopole flux as,

$$
F_{m}^{\prime} \simeq \frac{\rho_{\mathrm{DM}}^{(\text {local })}}{\rho_{\mathrm{DM}}} F_{m}
$$

where we have roughly estimated the local monopole enhancement as $\rho_{\mathrm{DM}}^{\text {(local) }} / \rho_{\mathrm{DM}} \sim 10^{5}$, with $\rho_{\mathrm{DM}}^{\text {(local) }} \simeq$ $0.3 \mathrm{GeV} / \mathrm{cm}^{3}$ the local dark matter halo density, $\rho_{\text {DM }} \simeq$ $1.2 \times 10^{-6} \mathrm{GeV} / \mathrm{cm}^{3}$ the average dark matter density in the Universe, and $F_{m}$ the flux if the GUT monopole were uniformly distributed throughout the Universe. The upper bounds on the parameter space set by $F_{m}^{\prime} \lesssim 10^{-18} \sim$ $10^{-28} \mathrm{~cm}^{-2} \mathrm{~s}^{-1}$ are shown in Fig. 1 with orange bands that rule out the regions below them. In most cases, the bound from the GUT monopole is weaker than that from the intermediate scale monopole. The exception only occurs when $M_{S} \sim 10^{4} \mathrm{GeV}$ with threshold effects included for the RG evolution.

If the $G_{3221} \rightarrow G_{\mathrm{SM}} \times M$ phase transition is of the strong first-order and experiences a supercooling phase, produced monopoles are diluted and the monopole density is suppressed. In this case, the constraint discussed above is

\footnotetext{
${ }^{8}$ The intermediate scale monopoles from the breaking of $G_{422}$ do not catalyze these processes.
} 
irrelevant. The abundance of cosmic strings produced at such a first order phase transition may be different from that of a second-order transition. However, it hardly affects the GW generation because it has been known that a network of cosmic strings reaches a scaling solution [93].

\section{DISCUSSIONS}

For a given mass spectrum of supersymmetric particles, precise gauge coupling unification relates the intermediate scale $M_{R}$ of cosmic strings to the $S O(10)$ breaking scale $M_{X}$ or the size of the unified gauge coupling $\alpha_{U}$. In Fig. 1, we compare the two extreme cases of the universal soft masses $M_{S}$ (left panel) and the split SUSY spectrum with gaugino masses fixed at $\mathcal{O}(1) \mathrm{TeV}$ while the other supersymmetric particles have a universal mass $M_{S}$ (right panel). Since we do not expect large modifications to these results when we take account of the detailed spectrum beyond the universal mass, these results demonstrate the spectrum dependence of the gauge unification conditions. It is worth noting that the GUT-scale threshold corrections can play important roles. As discussed in Appendix B, the size of the corrections highly depends on the precise mass spectrum of the GUT-scale particles. For a demonstration purpose, we pick up two reference points and compare results in the left and middle panels of Fig. 1.

The high cosmic string scale $M_{R} \gtrsim 10^{14} \mathrm{GeV}$ inferred by the NANOGrav signal is a generic feature of the SUSY $S O$ (10) model as we demonstrate in Fig. 1. The left panel of Fig. 1, which corresponds to the universal soft masses, shows that the current constraint on the proton lifetime and also the monopole overproduction constraint require supersymmetric particles at a low-energy scale under the condition of the precise gauge coupling unification without the GUT-scale threshold corrections. There is a tiny allowed parameter region with $M_{S}=\mathcal{O}(10) \mathrm{TeV}$ and $M_{R}=$ $\mathcal{O}\left(10^{15}\right) \mathrm{GeV}$ which is consistent with the NANOGrav data. However, this region with $\tan \beta \lesssim 3$ is in tension with the observed SM Higgs mass [94]. As suggested in the middle panel, the GUT-scale threshold corrections can change this conclusion completely. The GUT scale $M_{X}$ tends to be higher, which renders the $p \rightarrow \pi^{0} e^{+}$decay unobservable unless $\alpha_{U}=\mathcal{O}(1)$. There is a vast parameter region unconstrained by current and future proton decay experiments. In this case, the GW observation together with a better understanding of cosmic string formation serves as the only means to narrow down the scale $M_{R}$ and thus the $S O(10)$ breaking scale $M_{X}$ and the size of the unified gauge coupling $\alpha_{U}$ through the requirement of gauge coupling unification. For the case of split SUSY without threshold correction, the right panel of Fig. 1 also shows a large allowed region with $M_{S} \gtrsim 10 \mathrm{TeV}$, which can be consistent with the SM Higgs mass while explaining the stochastic GW signal. The search for the proton decay at the HyperKamiokande will explore a large fraction of the allowed parameter space, and the decay will be observed when
$M_{R}=\mathcal{O}\left(10^{14}\right) \mathrm{GeV}$, which is favored by the recent numerical simulation of cosmic strings to explain the NANOGrav data [32], with a moderate choice of $M_{S}$. Because of the high cosmic string scale, the monopole abundance also places nontrivial constraints for the low $\alpha_{U}$ region, even if its initial density is diluted by inflation. This complements the proton decay constraints at the high $\alpha_{U}$ region. In conclusion, the GW observation gives us a way to probe the supersymmetric grand unification. To extract the intermediate scale $M_{R}$ from the GW signal precisely, it is essential to reduce the uncertainty for the initial loop size $\alpha$ in the cosmic string spectrum.

\section{ACKNOWLEDGMENTS}

S. C. is grateful to Ofri Telem for useful comments and discussions. Y.N. would like to thank Kohei Fujikura, Motoo Suzuki and Tsutomu T. Yanagida for discussions. Y. N. is grateful to Kavli IPMU for their hospitality during the COVID-19 pandemic. J.Z. would like to thank Goran Senjanovic for pedagogical conversations on SUSY $S O(10)$ during his visit to TDLI in January. S. C. is supported by JSPS KAKENHI grant. J.Z. is supported in part by the National NSF of China under Grants No. 11675086 and No. 11835005. S. C. is supported by the Director, Office of Science, Office of High Energy Physics of the U.S. Department of Energy under the Contract No. DE-AC02-05CH1123.

\section{APPENDIX A: ONE INTERMEDIATE SCALE}

The simplest possibility of the multistep $S O(10)$ breaking is the case with one intermediate scale, $S O(10) \rightarrow H \rightarrow G_{\mathrm{SM}} \times M$. We focus on models with $H=G_{3221}$ or $G_{421}$ where cosmic strings are formed at the intermediate scale $M_{R}$ as shown in Table I.

The hierarchical VEVs, $\quad|s| \sim|a| \sim M_{X} \gg|\sigma| \sim$ $M_{R} \gg|b| \sim M_{2}=M_{R}^{2} / M_{X}$, lead to the breaking pattern of $S O(10) \rightarrow G_{3221} \rightarrow G_{\mathrm{SM}} \times M$. The mass spectrum of this model is obtained by taking the limit of $M_{C} \rightarrow M_{X}$ in Table II. As in the case of two intermediate scales, the gauge couplings of $S U(2)_{R}$ and $U(1)_{B-L}$ are matched to that of the SM hypercharge at $M_{R}$ through Eq. (4). All the gauge couplings of $G_{3221}$ run further to the unification scale $M_{X}$ and unify into a unique value $\alpha_{U}$. Consequently, we obtain the one-loop level relationship between the gauge coupling constants $\alpha_{i}$ at $M_{Z}$ and $\alpha_{U}$ as

$$
\begin{aligned}
\frac{2 \pi}{\alpha_{i}\left(M_{Z}\right)}= & \frac{2 \pi}{\alpha_{U}}+\left[b_{i}^{(1)} \ln \frac{M_{S}}{M_{Z}}+b_{i}^{(2)} \ln \frac{M_{2}}{M_{S}}\right. \\
& \left.+b_{i}^{(a)} \ln \frac{M_{R}}{M_{2}}+b_{i}^{(b)} \ln \frac{M_{X}}{M_{R}}\right],
\end{aligned}
$$

where $b_{i}^{(1)}$ and $b_{i}^{(2)}$ are given in Table III, while $b_{i}^{(a)}=$ $(57 / 5,1,-3)$ and $b_{i}^{(b)}=(9,1,-3)$. We solve the three 
equations (A1) in terms of the three parameters $M_{R}, M_{X}$, and $\alpha_{U}$, and obtain a set of solutions as a function of $M_{S}$. It is found that the correct hierarchy $M_{Z}<M_{S}<M_{R}<M_{X}$ requires $M_{S} \lesssim 1 \mathrm{TeV}$, which is already excluded by collider searches. GUT threshold corrections do not change our conclusion.

The breaking pattern of $S O(10) \rightarrow G_{421} \rightarrow G_{\mathrm{SM}} \times M$ is realized by the hierarchical VEVs, $|s| \sim|b| \sim M_{X} \gg|\sigma| \sim$ $M_{R} \gg|a| \sim M_{2}$. In this setup, there are light degrees of freedom described as $(6,1)_{ \pm 4 / 3}$ and $(1,1)_{ \pm 0}$ under $G_{\mathrm{SM}}$, all of which have masses of $\mathcal{O}\left(M_{2}\right)$, in addition to the to-be Nambu-Goldstone bosons with masses of $\mathcal{O}\left(M_{R}\right)$. At the intermediate scale $M_{R}$, the matching between the gauge coupling constants is given by

$$
\begin{gathered}
\alpha_{4}\left(M_{R}\right)=\alpha_{3}\left(M_{R}\right), \\
\frac{2}{5} \alpha_{4}^{-1}\left(M_{R}\right)+\frac{3}{5} \alpha_{1 R}^{-1}\left(M_{R}\right)=\alpha_{1}^{-1}\left(M_{R}\right),
\end{gathered}
$$

where $\alpha_{1 R}$ denotes the $U(1)_{R}$ gauge coupling. The RG evolution of the gauge couplings is again governed by Eq. (A1), though in this case $b_{i}^{(a)}=(97 / 5,1,2)$ and $b_{i}^{(b)}=(81 / 5,1,0)$. Again we found that the hierarchy $M_{Z}<M_{S}<M_{R}<M_{X}$ requires $M_{S}<1 \mathrm{TeV}$, which is already excluded.

\section{APPENDIX B: THRESHOLD CORRECTIONS}

We here estimate threshold corrections to the couplings at the GUT scale $M_{X}$ from the spectrum of $S, A, \Sigma$, and $\bar{\Sigma}$. If we ignore 10, the theory is defined by the terms in Eq. (2). Applying the vacuum conditions, the mass parameters $m_{S}$, $m_{A}$, and $m_{\Sigma}$ can be traded with the VEVs $s, a$, and $\sigma$. The remaining free parameters are the couplings $\lambda, \lambda_{S}, \eta_{S}, \bar{\eta}_{S}$, and $\eta_{A}$. The threshold corrections can then be parametrized by these dimensionless couplings and the VEVs. The oneloop contribution to the running coupling $\alpha_{i}^{-1}(Q)$ at $Q \gtrsim$ $M_{X}$ from all chiral superfields with the same $G_{\mathrm{SM}}$ representation $R$ is $2 \pi \Delta \alpha_{i}^{-1}(Q)=\sum_{j} b_{i}^{R} \ln \frac{m_{j}}{Q} . b_{i}^{R}=l_{i}^{R}$ is the
Dynkin index of the representation $R$. Since these superfields have the same SM quantum number and mix with each other, their mass terms are generally described by a nondiagonal mass matrix $M(R)$ after intermediate symmetry breakings, as given in the Appendix of [64]. Neglecting Nambu-Goldstone bosons, ${ }^{9}$ the contribution can be evaluated as

$$
2 \pi \Delta \alpha_{i}^{-1}(Q)=b_{i}^{R} \ln \left|\frac{a_{k}(M(R))}{Q^{n-k}}\right|,
$$

where $n$ is the dimension of the mass matrix $M(R), k$ is the number of zero eigenvalues that correspond to the NambuGoldstone bosons, and $a_{k}(M(R))$ is the coefficient of the $x^{k}$ term of the characteristic polynomial $|\operatorname{Det}(M(R)-x \mathbf{1})|$. For comparison, the 1-loop stepwise contribution to the running coupling is $2 \pi \Delta_{0} \alpha_{i}^{-1}(Q)=\sum_{j} b_{i}^{R} \ln \frac{\tilde{Q}_{j}}{Q}$, where $\tilde{Q}_{j} \in\left\{M_{1}, M_{2}, M_{R}, M_{C}, M_{X}\right\}$ is the mass scale of the particle $j$. The threshold correction $\lambda_{i} \equiv$ $2 \pi \sum_{R}\left(\Delta \alpha_{i}^{-1}(Q)-\Delta_{0} \alpha_{i}^{-1}(Q)\right)$ is then,

$$
\lambda_{i}=\sum_{R} b_{i}^{R} \ln \left|\frac{a_{k}(M(R))}{\Pi_{j \neq \mathrm{NG}} \tilde{Q}_{j}}\right|,
$$

with NG stands for Nambu-Goldstone bosons. For gauge coupling unification, it is more convenient to calculate

$$
\Delta \lambda_{i j} \equiv \lambda_{i}-\lambda_{j}
$$

Given the mass matrices in [64], the calculation $\Delta \lambda_{i j}$ is straightforward with Eq. (B2). For our model A, identifying $M_{X}=s, M_{C}=a, M_{R}=\sigma$, and in the limit of $s \gg a \gg \sigma$, we obtain

$$
\Delta \lambda_{12} \simeq-7.4-\frac{2}{5}\left(16 \ln \lambda-5 \ln \eta_{A}-2 \ln \xi_{S}^{3}\right),
$$

and

$$
\Delta \lambda_{13} \simeq \begin{cases}-9.0-\frac{3}{5}\left(4 \ln \lambda-5 \ln \eta_{A}+2 \ln \xi_{S}^{3}\right), & \text { for } \sigma^{2} / a>a^{2} / s, \\ -9.0+\ln \frac{a^{3}}{s \sigma^{2}}-\frac{3}{5}\left(4 \ln \lambda-5 \ln \eta_{A}+2 \ln \xi_{S}^{3}\right), & \text { for } \sigma^{2} / a<a^{2} / s,\end{cases}
$$

where we have defined $\xi_{S}^{3} \equiv \eta_{S} \bar{\eta}_{S} \lambda_{S}$. In the middle panel of Fig. 1, we define the gauge coupling at the GUT scale as

$$
\begin{gathered}
\alpha_{1}\left(M_{X}\right)=\alpha_{U}, \\
\alpha_{i}\left(M_{X}\right)=\alpha_{U}\left(1+\frac{\alpha_{U}}{2 \pi} \Delta \lambda_{1 i}\right) \quad(i=2,3),
\end{gathered}
$$

with $\Delta \lambda_{12}=-7.5$ and $\Delta \lambda_{13}=-9.0$, which are typical values obtained when all the dimensionless couplings are equal to unity.

\footnotetext{
${ }^{9}$ The symmetry breaking scale is defined as the mass of gauge bosons so Nambu-Goldstone bosons do not contribute to the threshold correction.
} 
[1] H. Georgi and S. Glashow, Unity of All Elementary Particle Forces, Phys. Rev. Lett. 32, 438 (1974).

[2] T. Yanagida, Horizontal symmetry and masses of neutrinos, Prog. Theor. Phys. 64, 1103 (1980).

[3] P. Minkowski, $\mu \rightarrow e \gamma$ at a rate of one out of $10^{9}$ muon decays?, Phys. Lett. 67B, 421 (1977).

[4] M. Gell-Mann, P. Ramond, and R. Slansky, Complex spinors and unified theories, Conf. Proc. C 790927, 315 (1979).

[5] H. Fritzsch and P. Minkowski, Unified interactions of leptons and hadrons, Ann. Phys. (N.Y.) 93, 193 (1975).

[6] H. Georgi, The state of the art-Gauge theories, AIP Conf. Proc. 23, 575 (1975).

[7] L. M. Krauss and F. Wilczek, Discrete Gauge Symmetry in Continuum Theories, Phys. Rev. Lett. 62, 1221 (1989).

[8] L. E. Ibanez and G. G. Ross, Discrete gauge symmetry anomalies, Phys. Lett. B 260, 291 (1991).

[9] L. E. Ibanez and G. G. Ross, Discrete gauge symmetries and the origin of baryon and lepton number conservation in supersymmetric versions of the standard model, Nucl. Phys. B368, 3 (1992).

[10] S. P. Martin, Some simple criteria for gauged R-parity, Phys. Rev. D 46, R2769 (1992).

[11] H. Goldberg, Constraint on the Photino Mass from Cosmology, Phys. Rev. Lett. 50, 1419 (1983); 103, 099905(E) (2009).

[12] J. R. Ellis, J. Hagelin, D. V. Nanopoulos, K. A. Olive, and M. Srednicki, Supersymmetric relics from the big bang, Nucl. Phys. B238, 453 (1984).

[13] K. Abe et al. (Super-Kamiokande Collaboration), Search for proton decay via $p \rightarrow e^{+} \pi^{0}$ and $p \rightarrow \mu^{+} \pi^{0}$ in 0.31 megaton years exposure of the Super-Kamiokande water Cherenkov detector, Phys. Rev. D 95, 012004 (2017).

[14] K. Abe et al. (Super-Kamiokande Collaboration), Search for proton decay via $p \rightarrow \nu K^{+}$using 260 kiloton · year data of Super-Kamiokande, Phys. Rev. D 90, 072005 (2014).

[15] K. Abe et al. (Hyper-Kamiokande Collaboration), Hyperkamiokande design report, arXiv:1805.04163.

[16] R. Acciarri et al. (DUNE Collaboration), Longbaseline neutrino facility (LBNF) and deep underground neutrino experiment (DUNE): Conceptual design report, volume 2: The physics program for DUNE at LBNF, arXiv:1512.06148.

[17] F. An et al. (JUNO Collaboration), Neutrino physics with JUNO, J. Phys. G 43, 030401 (2016).

[18] T. Kibble, Topology of cosmic domains and strings, J. Phys. A 9, 1387 (1976).

[19] T. Kibble, G. Lazarides, and Q. Shafi, Strings in SO(10), Phys. Lett. 113B, 237 (1982).

[20] R. Jeannerot, J. Rocher, and M. Sakellariadou, How generic is cosmic string formation in SUSY GUTs, Phys. Rev. D 68, 103514 (2003).

[21] A. Vilenkin, Gravitational radiation from cosmic strings, Phys. Lett. 107B, 47 (1981).

[22] T. Vachaspati and A. Vilenkin, Gravitational radiation from cosmic strings, Phys. Rev. D 31, 3052 (1985).

[23] C. Ringeval, M. Sakellariadou, and F. Bouchet, Cosmological evolution of cosmic string loops, J. Cosmol. Astropart. Phys. 02 (2007) 023.
[24] J. J. Blanco-Pillado, K. D. Olum, and B. Shlaer, Large parallel cosmic string simulations: New results on loop production, Phys. Rev. D 83, 083514 (2011).

[25] M. Hindmarsh, Signals of inflationary models with cosmic strings, Prog. Theor. Phys. Suppl. 190, 197 (2011).

[26] W. Buchmüller, V. Domcke, K. Kamada, and K. Schmitz, The gravitational wave spectrum from cosmological $B-L$ breaking, J. Cosmol. Astropart. Phys. 10 (2013) 003.

[27] D. Matsunami, L. Pogosian, A. Saurabh, and T. Vachaspati, Decay of Cosmic String Loops Due to Particle Radiation, Phys. Rev. Lett. 122, 201301 (2019).

[28] Z. Arzoumanian et al. (NANOGrav Collaboration), The NANOGrav 12.5-year data set: Search for an isotropic stochastic gravitational-wave background, Astrophys. J. Lett. 905, L34 (2020).

[29] L. Lentati et al., European pulsar timing array limits on an isotropic stochastic gravitational-wave background, Mon. Not. R. Astron. Soc. 453, 2576 (2015).

[30] R. M. Shannon et al., Gravitational waves from binary supermassive black holes missing in pulsar observations, Science 349, 1522 (2015).

[31] G. Lazarides, R. Maji, and Q. Shafi, NANOGrav and PPTA tension: Gravity waves, cosmic strings, and inflation, arXiv:2104.02016.

[32] J. Ellis and M. Lewicki, Cosmic String Interpretation of NANOGrav Pulsar Timing Data, Phys. Rev. Lett. 126, 041304 (2021).

[33] S. Blasi, V. Brdar, and K. Schmitz, Has NANOGrav Found First Evidence for Cosmic Strings?, Phys. Rev. Lett. 126, 041305 (2021).

[34] W. Buchmuller, V. Domcke, and K. Schmitz, From NANOGrav to LIGO with metastable cosmic strings, Phys. Lett. B 811, 135914 (2020).

[35] R. Samanta and S. Datta, Gravitational wave complementarity and impact of NANOGrav data on gravitational leptogenesis: Cosmic strings, J. High Energy Phys. 05 (2021) 211.

[36] Y. Nakai, M. Suzuki, F. Takahashi, and M. Yamada, Gravitational waves and dark radiation from dark phase transition: Connecting NANOGrav pulsar timing data and Hubble tension, Phys. Lett. B 816, 136238 (2021).

[37] A. Addazi, Y.-F. Cai, Q. Gan, A. Marciano, and K. Zeng, NANOGrav results and dark first order phase transitions, Sci. China Phys. Mech. Astron. 64, 290411 (2021).

[38] W. Ratzinger and P. Schwaller, Whispers from the dark side: Confronting light new physics with NANOGrav data, SciPost Phys. 10, 047 (2021).

[39] A. Neronov, A. Roper Pol, C. Caprini, and D. Semikoz, NANOGrav signal from MHD turbulence at QCD phase transition in the early universe, Phys. Rev. D 103, L041302 (2021).

[40] L. Bian, J. Liu, and R. Zhou, NanoGrav 12.5-yr data and different stochastic Gravitational wave background sources, Phys. Rev. D 103, L081301 (2021).

[41] V. Vaskonen and H. Veermäe, Did NANOGrav see a Signal from Primordial Black Hole Formation?, Phys. Rev. Lett. 126, 051303 (2021).

[42] V. De Luca, G. Franciolini, and A. Riotto, NANOGrav Hints to Primordial Black Holes as Dark Matter, Phys. Rev. Lett. 126, 041303 (2021). 
[43] K. Kohri and T. Terada, Solar-mass primordial black holes explain NANOGrav hint of gravitational waves, Phys. Lett. B 813, 136040 (2021).

[44] S. Sugiyama, V. Takhistov, E. Vitagliano, A. Kusenko, M. Sasaki, and M. Takada, Testing stochastic gravitational wave signals from primordial black holes with optical telescopes, Phys. Lett. B 814, 136097 (2021).

[45] G. Domènech and S. Pi, NANOGrav hints on planet-mass primordial black holes, arXiv:2010.03976.

[46] K. Inomata, M. Kawasaki, K. Mukaida, and T. T. Yanagida, NANOGrav Results and LIGO-Virgo Primordial Black Holes in Axion-Like Curvaton Model, Phys. Rev. Lett. 126, 131301 (2021).

[47] R. Namba and M. Suzuki, Implications of gravitationalwave production from dark photon resonance to pulsartiming observations and effective number of relativistic species, Phys. Rev. D 102, 123527 (2020).

[48] J. J. Blanco-Pillado, K. D. Olum, and B. Shlaer, The number of cosmic string loops, Phys. Rev. D 89, 023512 (2014).

[49] J. J. Blanco-Pillado and K. D. Olum, Stochastic gravitational wave background from smoothed cosmic string loops, Phys. Rev. D 96, 104046 (2017).

[50] M. Fukugita and T. Yanagida, Physics of Neutrinos and Applications to Astrophysics (Springer, 2003), https:// doi.org/10.1007/978-3-662-05119-1.

[51] J. A. Dror, T. Hiramatsu, K. Kohri, H. Murayama, and G. White, Testing the Seesaw Mechanism and Leptogenesis with Gravitational Waves, Phys. Rev. Lett. 124, 041804 (2020).

[52] W. Buchmuller, V. Domcke, H. Murayama, and K. Schmitz, Probing the scale of grand unification with gravitational waves, Phys. Lett. B 809, 135764 (2020).

[53] S. F. King, S. Pascoli, J. Turner, and Y.-L. Zhou, Gravitational Waves and Proton Decay: Complementary Windows into GUTs, Phys. Rev. Lett. 126, 021802 (2021).

[54] J. Chakrabortty, G. Lazarides, R. Maji, and Q. Shafi, Primordial monopoles and strings, inflation, and gravity waves, J. High Energy Phys. 02 (2021) 114.

[55] B. Bajc, A. Melfo, G. Senjanovic, and F. Vissani, The minimal supersymmetric grand unified theory. 1. Symmetry breaking and the particle spectrum, Phys. Rev. D 70, 035007 (2004).

[56] C.S. Aulakh, B. Bajc, A. Melfo, G. Senjanovic, and F. Vissani, The minimal supersymmetric grand unified theory, Phys. Lett. B 588, 196 (2004).

[57] C. S. Aulakh, B. Bajc, A. Melfo, A. Rasin, and G. Senjanovic, SO(10) theory of R-parity and neutrino mass, Nucl. Phys. B597, 89 (2001).

[58] S. Weinberg, Effective gauge theories, Phys. Lett. 91B, 51 (1980).

[59] L. J. Hall, Grand unification of effective gauge theories, Nucl. Phys. B178, 75 (1981).

[60] B. Bajc, A. Melfo, G. Senjanovic, and F. Vissani, Yukawa sector in non-supersymmetric renormalizable SO(10), Phys. Rev. D 73, 055001 (2006).

[61] J. R. Ellis and M. K. Gaillard, Fermion masses and Higgs representations in SU(5), Phys. Lett. 88B, 315 (1979).

[62] C. Balazs, T. Li, D. V. Nanopoulos, and F. Wang, Realistic standard model fermion mass relations in generalized minimal supergravity (GmSUGRA), J. High Energy Phys. 02 (2011) 096.

[63] G. Anderson, S. Raby, S. Dimopoulos, L. J. Hall, and G. D. Starkman, A Systematic SO(10) operator analysis for fermion masses, Phys. Rev. D 49, 3660 (1994).

[64] A. Melfo, A. Ramirez, and G. Senjanovic, Type II see-saw dominance in SO(10), Phys. Rev. D 82, 075014 (2010).

[65] H. Arason, D. J. Castano, B. Keszthelyi, S. Mikaelian, E. J. Piard, P. Ramond, and B. D. Wright, Renormalization group study of the standard model and its extensions. 1. The Standard model, Phys. Rev. D 46, 3945 (1992).

[66] S. P. Martin and M. T. Vaughn, Two loop renormalization group equations for soft supersymmetry breaking couplings, Phys. Rev. D 50, 2282 (1994); 78, 039903(E) (2008).

[67] N. Sakai and T. Yanagida, Proton decay in a class of supersymmetric grand unified models, Nucl. Phys. B197, 533 (1982).

[68] S. Weinberg, Supersymmetry at ordinary energies. 1. Masses and conservation laws, Phys. Rev. D 26, 287 (1982).

[69] S. Dimopoulos, S. Raby, and F. Wilczek, Proton decay in supersymmetric models, Phys. Lett. 112B, 133 (1982).

[70] K. Babu, J. C. Pati, and F. Wilczek, Fermion masses, neutrino oscillations, and proton decay in the light of Super-Kamiokande, Nucl. Phys. B566, 33 (2000).

[71] H. Goh, R. Mohapatra, S. Nasri, and S.-P. Ng, Proton decay in a minimal SUSY SO(10) model for neutrino mixings, Phys. Lett. B 587, 105 (2004).

[72] J. Hisano, D. Kobayashi, T. Kuwahara, and N. Nagata, Decoupling can revive minimal supersymmetric SU(5), J. High Energy Phys. 07 (2013) 038.

[73] S. Weinberg, Baryon and Lepton Nonconserving Processes, Phys. Rev. Lett. 43, 1566 (1979).

[74] P. Langacker, Grand unified theories and proton decay, Phys. Rep. 72, 185 (1981).

[75] P. Nath and P. F. Perez, Proton stability in grand unified theories, in strings and in branes, Phys. Rep. 441, 191 (2007).

[76] J. Hisano, D. Kobayashi, Y. Muramatsu, and N. Nagata, Two-loop renormalization factors of dimension-six proton decay operators in the supersymmetric standard models, Phys. Lett. B 724, 283 (2013).

[77] G. Lazarides and Q. Shafi, The fate of primordial magnetic monopoles, Phys. Lett. 94B, 149 (1980).

[78] J. Preskill, Magnetic monopoles, Annu. Rev. Nucl. Part. Sci. 34, 461 (1984).

[79] J. Preskill, Cosmological Production of Superheavy Magnetic Monopoles, Phys. Rev. Lett. 43, 1365 (1979).

[80] M. S. Turner, Thermal production of superheavy magnetic monopoles in the early universe, Phys. Lett. 115B, 95 (1982).

[81] M. Ambrosio et al. (MACRO Collaboration), Final results of magnetic monopole searches with the MACRO experiment, Eur. Phys. J. C 25, 511 (2002).

[82] E. N. Parker, The origin of magnetic fields, Astrophys. J. 160, 383 (1970). 
[83] P. Zyla et al. (Particle Data Group Collaboration), Review of particle physics, Prog. Theor. Exp. Phys. 2020, 083 C01 (2020).

[84] J. Callan and G. Curtis, Dyon-fermion dynamics, Phys. Rev. D 26, 2058 (1982).

[85] S. Dawson and A. Schellekens, Monopole catalysis of proton decay in $\mathrm{SO}(10)$ grand unified models, Phys. Rev. D 27, 2119 (1983).

[86] K. Freese, Do monopoles keep white dwarfs hot?, Astrophys. J. 286, 216 (1984).

[87] K. Freese and E. Krasteva, A bound on the flux of magnetic monopoles from catalysis of nucleon decay in white dwarfs, Phys. Rev. D 59, 063007 (1999).

[88] S. Dimopoulos, J. Preskill, and F. Wilczek, Catalyzed nucleon decay in neutron stars, Phys. Lett. 119B, 320 (1982).

[89] J. A. Harvey, Monopoles in neutron stars, Nucl. Phys. B236, 255 (1984).
[90] K. Freese, M. S. Turner, and D. N. Schramm, Monopole Catalysis of Nucleon Decay in Old Pulsars, Phys. Rev. Lett. 51, 1625 (1983).

[91] E. W. Kolb and M. S. Turner, Limits from the soft x-ray background on the temperature of old neutron stars and on the flux of superheavy magnetic monopoles, Astrophys. J. 286, 702 (1984).

[92] J. A. Harvey, M. A. Ruderman, and J. Shaham, Effects of neutron star superconductivity on magnetic monopoles and core field decay, Phys. Rev. D 33, 2084 (1986).

[93] T. Kibble, Evolution of a system of cosmic strings, Nucl. Phys. B252, 227 (1985); 261, 750(E) (1985).

[94] E. Bagnaschi, G. F. Giudice, P. Slavich, and A. Strumia, Higgs mass and unnatural supersymmetry, J. High Energy Phys. 09 (2014) 092. 